\title{
Contaminación y salud pública en hábitats urbanos: metales pesados en sedimentos viales de Bogotá, Colombia
}

\author{
CARlos Zafra-Mejía ${ }^{1}$ \\ JULio BELTRÁN-VARGAS ${ }^{2}$ \\ YOLANDA HERNÁNDEZ-PEÑA ${ }^{2}$
}

\section{Resumen}

Los organismos de investigación en urbanismo, ambiente y salud pública reportan el interés creciente de estudiar la contaminación por metales pesados (MP) en hábitats urbanos. Se presenta un estudio observacional descriptivo de las concentraciones de MP en sedimentos viales de un hábitat urbano latinoamericano: Bogotá-Colombia. Existió coincidencia entre las concentraciones de MP reportadas a nivel mundial y las observadas en Bogotá. Las concentraciones en Bogotá tendieron a ser inferiores a las reportadas en ciudades de Norteamérica-Europa-Asía. El elemento más crítico fue el Pb. Este metal superó entre 1,02-4,30 veces el límite legislativo de referencia más exigente por ingestión, inhalación y contacto dérmico (Cataluña-España: $60 \mathrm{mg} / \mathrm{kg}$ ). Durante periodos climáticos de tiempo seco se observaron incrementos en las concentraciones de MP, entre 1,31-2,11 veces. Los hallazgos se constituyen en un punto de referencia durante el desarrollo de estrategias para la protección de la salud pública y el ambiente en hábitats urbanos.

Palabras clave: hábitats urbanos, metales pesados, salud pública.

\section{Pollution And Public Health In Urban Habitats: Heavy Metals In Road Sediments Of Bogota, Colombia}

\begin{abstract}
Research agencies in urban planning, environment, and public health report the growing interest of studying the pollution by heavy metals (HMs) in urban habitats. A descriptive observational study of the HM concentrations in road sediments of an urban habitat in Latin America is displayed: Bogota-Colombia. There was a coincidence between the HM

\footnotetext{
Grupo de Investigación en Ingeniería Ambiental, Universidad Distrital Francisco José de Caldas, Bogotá, Colombia.
}

2 Universidad Distrital Francisco José de Caldas, Bogotá, Colombia.
\end{abstract}

Autor de correspondencia: Zafra-Mejía C. (Carlos): Facultad de Medio Ambiente y Recursos Naturales, Universidad Distrital Francisco José de Caldas, Carrera 5 Este № 15 82, Bogotá, Colombia. Tel.: (1) 3239300, ext. 4040. Correo electrónico:czafra@udistrital.edu.co
Historia del artículo:

Artículo recibido: 29-V-2018 / Aprobado: 15-I-2020

Disponible online: 15 de enero de 2020

Discusión abierta hasta septiembre de 2021 
concentrations reported worldwide and those observed in Bogota. Concentrations in Bogota tended to be lower than those reported in cities of North America, Europe, and Asia. The most critical element was $\mathrm{Pb}$. The concentrations of $\mathrm{Pb}$ exceeded between 1.02-4.30 times the legislative limit most demanding for ingestion, inhalation, and dermal contact (Catalonia-Spain: $60 \mathrm{mg} / \mathrm{kg}$ ). During climatic periods of dry time there were increases in HM concentrations (between 1.31-2.11 times). The findings are a point of reference during the development of strategies for the protection of the public health and environment in urban habitats.

Keywords: urban habitats, heavy metals, public health.

\section{contaminação e saúde pública em habitats urbanos: metais pesados em sedimentos viales de Bogotá, Colômbia}

\section{Resumo}

As organizações de pesquisa em planejamento urbano, meio ambiente e saúde pública relatam o crescente interesse em estudar a contaminação por metais pesados (MP) em habitats urbanos. Um estudo observacional descritivo das concentrações de MP em sedimentos rodoviários de um habitat urbano latino-americano é apresentado: Bogotá-Colômbia. Houve uma coincidência entre as concentrações de MP relatadas em todo o mundo e as observadas em Bogotá. As concentrações em Bogotá tendem a ser menores do que as relatadas em cidades da América do Norte, Europa e Ásia. 0 elemento mais crítico foi o $\mathrm{Pb}$. Este metal excedeu entre 1,02-4,30 vezes o limite legislativo de referência mais exigente para ingestão, inalação e contato dérmico (Catalunha-Espanha: $60 \mathrm{mg} / \mathrm{kg}$ ). Durante os períodos climáticos de clima seco, foram observados aumentos nas concentrações de MP, entre 1,31-2,11 vezes. Os achados constituem um ponto de referência durante o desenvolvimento de estratégias para a proteção da saúde pública e do meio ambiente em habitats urbanos.

Palavras-chave:mhabitats urbanos, metais pesados, saúde pública.

\section{Introducción}

La capacidad de nuestras sociedades para alcanzar y mantener en el tiempo unas condiciones habitacionales coherentes con el derecho a un hábitat digno, está inevitablemente vinculada a los mecanismos de generación de nuestras viviendas, barrios y ciudades, pues depende directamente de los recursos disponibles y de la capacidad de emisión de residuos (Arcas-Abella et al., 2011). En relación a este último aspecto, los organismos de investigación mundial en urbanismo, ambiente y salud pública reportan el interés creciente de estudiar la contaminación por metales pesados en hábitats urbanos, debido a su continua emisión, los largos periodos de residencia en el ambiente, el transporte dentro de las masas de aire, su deposición sobre la superficie, y a que son significativamente móviles y potencialmente biodisponibles (Qing et al., 2015).
La ciudad de Bogotá (Colombia) fue reconocida como el centro urbano de mayor densidad poblacional en Latinoamérica (26000 habitantes $/ \mathrm{km}^{2}$ en promedio), estando organizada en 20 localidades con un alto nivel de segregación socio-espacial y una tendencia creciente de la informalidad laboral; como resultado de esto, el ambiente urbano se ha deteriorado significativamente durante la última década (Sarmiento et al., 2015). Al respecto, la ciudad fue reportada por la Organización Panamericana de la Salud como el tercer hábitat urbano de mayor contaminación atmosférica en América Latina (Nelson et al., 2005).

Los sedimentos depositados en tiempo seco sobre las superficies viales han sido utilizados como indicadores de la presencia de metales pesados en hábitats urbanos, ya que se constituyeron en la acumulación más reciente de contaminantes en una zona determinada 
(Apeagyei et al., 2011; Yuen et al., 2012). Es decir, fueron sumideros de metales pesados y otros compuestos que provinieron de diversas fuentes de contaminación (Liu et al., 2014). La principal ventaja del uso de sedimentos viales en investigaciones sobre urbanismo, ambiente y salud pública radicó en que éstos fueron utilizados como indicadores de la calidad del agua, aire y suelo debido a los procesos de transporte y dispersión a los cuales estuvieron sometidos en el hábitat urbano (Amato et al., 2013; Zafra et al., 2017): (i) deposición (v.g., suelo y superficies impermeables), (ii) remoción (escorrentía y viento), (iii) suspensión (viento y turbulencia del tráfico), e (iv) intercepción (vegetación y mobiliario urbano).

Los anteriores procesos permiten sugerir la posibilidad de ingestión, inhalación y contacto dérmico con metales pesados por parte de los ciudadanos y afectación sobre el medio natural urbano. Como los metales pesados son generalmente no biodegradables (acumulativos) y no se conocen sus mecanismos homeostáticos (equilibrio en un medio interno), es muy probable que elevadas concentraciones generen efectos negativos sobre la vida biológica (Vera et al., 2015). En seres humanos existe acumulación en tejidos grasos y el sistema circulatorio, efectos adversos sobre el sistema nervioso central y el funcionamiento de los órganos internos, y actúan como cofactores en enfermedades como el cáncer (Dockery y Pope, 1994).

El objetivo de este artículo es presentar un estudio observacional descriptivo de las concentraciones de metales pesados asociadas con el sedimento depositado sobre superficies viales de un hábitat urbano latinoamericano: Bogotá, Colombia. Adicionalmente, se investigan dos superficies viales de la localidad conurbana de Soacha. En este estudio se comparan las concentraciones locales de metales pesados con respecto a las reportadas por investigaciones en hábitats urbanos a nivel mundial. Se evalúa también el grado de contaminación en relación a legislación de referencia para la protección de la salud humana en suelo urbano. Finalmente, se estudian las concentraciones de metales pesados con respecto a periodos climáticos urbanos definidos por la variación de la precipitación. La investigación se fundamenta en los tres metales pesados más reportados por los estudios sobre sedimentos viales en hábitats urbanos a nivel mundial: Pb, Zn y Cu (Eriksson et al., 2007).

\section{Materiales y Métodos}

\subsection{Descripción del hábitat de estudio}

Se realizó un estudio observacional descriptivo. Los hábitats en investigación se localizaron sobre superficies viales de las localidades de Fontibón (Z1), Barrios Unidos (Z2), Kennedy (Z3) y Puente Aranda (Z4) en la ciudad de Bogotá (8,85 millones de habitantes para 2015), en el centro de Colombia (Tabla 1). Adicionalmente, se investigaron dos superficies viales de la localidad conurbana de Soacha (0,53 millones de habitantes para 2015); una en el corredor vial Autopista Sur (AA) y otra en el casco urbano de la localidad (SO). Todas las superficies viales fueron seleccionadas por su cercanía (entre 9 y $665 \mathrm{~m}$ ) a estaciones de la red de calidad del aire de la Ciudad de Bogotá (RMCAB) y de la Corporación Autónoma Regional de Cundinamarca (CAR) en Soacha (Figura 1). Estas localidades también fueron elegidas debido a que en los informes de calidad del aire para la ciudad de Bogotá fueron reportadas como de alta (Z3 > Z4 > Z1) y baja (Z2) contaminación por material particulado atmosférico (SDA, 2012). La información climatológica utilizada en el presente estudio fue obtenida de las redes de monitoreo RMCAB y CAR.

\subsection{Sistema de recolección del sedimento} vial

Las muestras de sedimento vial en los hábitats de estudio se tomaron en tiempo seco, a un costado del bordillo $(0,50 \mathrm{~m})$, a la misma hora, y durante un período de un año (08/05/2010-08/05/2011) para Z1, Z2, Z3 y Z4, y 127 días (07/01/2010-14/05/2010) para AA y SO. La superficie de muestreo tuvo un área de $0,50 \mathrm{~m}^{2}$ $(0,707 \mathrm{~m} \times 0,707 \mathrm{~m})$. Las dimensiones del área de recolección del sedimento vial se garantizaron colocando sobre la superficie un marco de madera de idénticas dimensiones que las del área de muestreo. Se controló el lugar de muestreo para evitar repetir y estar cerca de anteriores puntos de recolección del sedimento vial. Para la recolección del sedimento se utilizó una escobilla de fibras plásticas y un recogedor de mano (sistema de recolección en seco). La superficie de muestreo fue barrida ligeramente para evitar el desprendimiento de partículas pertenecientes al pavimento e intentando aplicar el mismo esfuerzo sobre la escobilla durante todo el período de investigación. 


\begin{tabular}{|c|c|c|c|c|c|c|}
\hline Característica & $\begin{array}{l}\text { Fontibón } \\
\quad \text { Z1 }\end{array}$ & $\begin{array}{l}\text { Barrios Unidos } \\
\quad \text { Z2 }\end{array}$ & $\begin{array}{l}\text { Kennedy } \\
\text { Z3 }\end{array}$ & $\begin{array}{l}\text { Puente Aranda } \\
\text { Z4 }\end{array}$ & $\begin{array}{c}\text { Autopista Sur } \\
\text { AA }\end{array}$ & $\begin{array}{l}\text { Soacha } \\
\text { SO }\end{array}$ \\
\hline Coordenadas & $\begin{array}{l}04^{\circ} 40^{\prime} 09^{\prime \prime} \mathrm{N} \\
74^{\circ} 08^{\prime} 33^{\prime \prime} \mathrm{O}\end{array}$ & $\begin{array}{l}4^{\circ} 39^{\prime} 36^{\prime \prime N} \\
74^{\circ} 4^{\prime} 42^{\prime \prime O}\end{array}$ & $\begin{array}{l}04^{\circ} 35^{\prime} 45^{\prime \prime N} \\
74^{\circ} 08^{\prime} 48^{\prime \prime} \mathrm{O}\end{array}$ & $\begin{array}{l}04^{\circ} 37^{\prime} 49^{\prime \prime} \mathrm{N} \\
74^{\circ} 07^{\prime} 06^{\prime \prime} \mathrm{O}\end{array}$ & $\begin{array}{l}4^{\circ} 33^{\prime} 04^{\prime \prime N} \\
74^{\circ} 14^{\prime} 22^{\prime \prime} \mathrm{O}\end{array}$ & $\begin{array}{r}4^{\circ} 35^{\prime} 05^{\prime \prime N} \\
74^{\circ} 13^{\prime} 12^{\prime \prime} \mathrm{O}\end{array}$ \\
\hline $\begin{array}{l}\text { Precipitación promedio } \\
\text { anual }(\mathrm{mm})\end{array}$ & 850 & 950 & 900 & 850 & 900 & 900 \\
\hline $\begin{array}{l}\text { Concentración promedio } \\
\text { anual de } \mathrm{PM}_{10}\left(\mu \mathrm{g} / \mathrm{m}^{3}\right)\end{array}$ & 52,1 & 37,5 & 78,5 & 55,5 & 47,9 & 58,5 \\
\hline $\begin{array}{l}\text { Densidad poblacional } \\
\text { (habitantes/ha) }\end{array}$ & 600 & 600 & 480 & 160 & $<25$ & 600 \\
\hline Uso del suelo $(*)$ & $\mathrm{I}-\mathrm{R}$ & $\mathrm{R}$ & $\mathrm{R}-\mathrm{I}$ & $\mathrm{I}-\mathrm{C}$ & I-RU & $\mathrm{R}$ \\
\hline $\begin{array}{l}\text { Tráfico promedio diario } \\
\text { (Vehículos/día) }\end{array}$ & 650 & 1600 & 12300 & 13500 & 40100 & 2750 \\
\hline $\begin{array}{c}\text { Composición del tráfico (\%) } \\
\text { Z1/Z2/Z3/Z4/AA/SO }\end{array}$ & \multicolumn{6}{|c|}{$\begin{array}{c}\text { Carros: } 93 / 81 / 77 / 83 / 62 / 65 ; \text { camiones ligeros: 5/16/4/3/7/0; camiones sin remolque: } 1 / 1 / 2 / 2 / 5 / 0 \text {; camiones } \\
\text { con remolque: 0/0/0/1/7/0; buses: 1/2/17/11/10/35 4/Suave-Ádo del tra sentidoe estudio en Puente } \\
\text { Aranada. }\end{array}$} \\
\hline
\end{tabular}

Figura 1. Localización de los hábitats de estudio en la ciudad de Bogotá y la conurbación de Soacha - Colombia (Google Mapas, 2017). Z1 = Fontibón, Z2 = Barrios Unidos, Z3 = Kennedy, Z4 = Puente Aranda, AA = Autopista Sur, y SO = Soacha

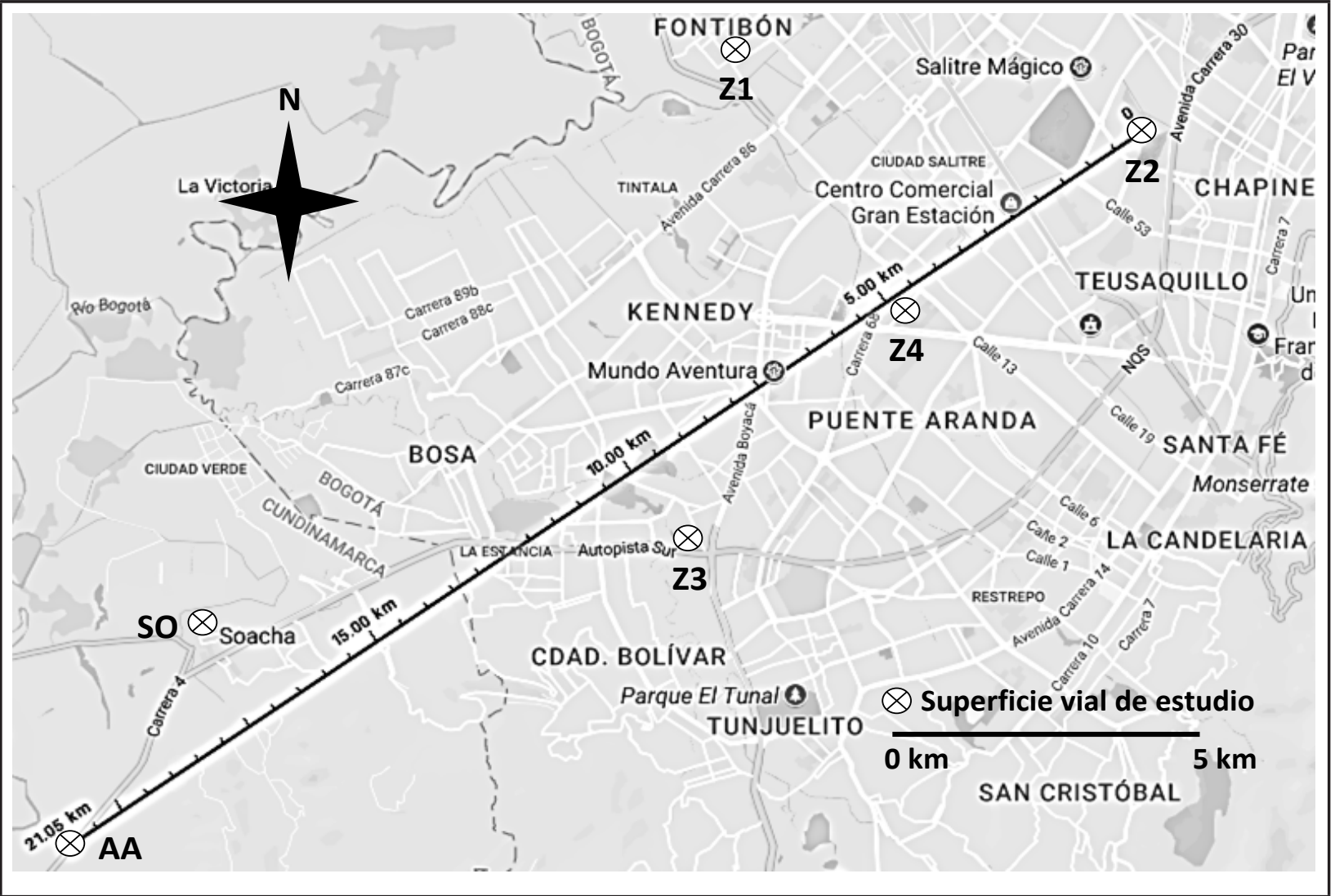


La frecuencia promedio de muestreo fue de 3 días en Soacha y 10 días en Bogotá. Estas variaciones en la frecuencia de muestreo se presentaron por la ocurrencia de eventos de precipitación que impidieron la recolección del sedimento vial en seco y, por lo tanto, impidieron la aplicación del sistema muestreo utilizado en el presente estudio. No obstante, estas variaciones en la frecuencia de los eventos de precipitación permitieron definir dos períodos climáticos de observación: (i) tiempo de lluvia, incremento en la frecuencia de las lluvias; y (ii) tiempo seco, disminución en la frecuencia de las lluvias. Estos períodos climáticos fueron verificados a partir de información del Instituto de Hidrología, Meteorología y Estudios Ambientales de Colombia (IDEAM), los cuales correspondieron al fenómeno climático de "La Niña-Oscilación del Sur" ocurrido durante el periodo de investigación (García et al., 2012). La cantidad de muestras recolectadas por cada zona de investigación en Bogotá y Soacha fue de 36 y 43, respectivamente; para un total de 144 muestras en Bogotá y 86 muestras en la conurbación de Soacha.

\subsection{Análisis de laboratorio}

La distribución por tamaños de las partículas del sedimento vial (<63 $\mu \mathrm{m}-250 \mu \mathrm{m})$ se determinó usando el método ISO-11277 (ISO, 2000). El análisis de la concentración de metales pesados en el sedimento se realizó para la fracción de tamaño inferior a $250 \mu \mathrm{m}$, debido a que las investigaciones han reportado que esta fracción de tamaño fue dominante en peso y tendió a registrar las mayores concentraciones de metales pesados en hábitats urbanos (Bian y Zhu, 2009; Zhao et al., 2010). La concentración de los metales pesados asociados con el sedimento vial se determinó por medio de espectrometría de absorción atómica con llama, ISO-11047 (ISO, 2000). Las muestras de sedimento fueron previamente digeridas en una mezcla de ácido clorhídrico y ácido nítrico (3:1; agua regia), método ISO-11466 (ISO, 2000). Los metales pesados analizados fueron $\mathrm{Pb}, \mathrm{Zn}$ y $\mathrm{Cu}$.

\subsection{Análisis estadístico}

La relación entre las variables de la matriz de revisión bibliográfica para las concentraciones de metales pesados (análisis comparativo mundial) fue evaluada mediante un análisis de conglomerados (clúster) utilizando el programa informático SPSS® versión 22.0. Los datos (variables) fueron estandarizados por medio de puntajes $\mathrm{Z}$ antes del análisis clúster, y se calcularon las distancias euclidianas de similitud entre las variables. Entonces se aplicó sobre los datos estandarizados un análisis jerárquico con el método de Ward y se desarrolló el correspondiente dendrograma. La distribución no normal de los datos fue determinada mediante la prueba estadística Shapiro-Wilk (Concentración de Pb: p-valor < 0,001, g.l. = 27; Zn: pvalor < 0,001, g.l. = 30; Cu: p-valor < 0,001, g.l. = 30). Se utilizaron estadísticos descriptivos, proporciones y la prueba de Mann-Whitney para profundizar el estudio entre las variables de la matriz de revisión bibliográfica desarrollada. Posteriormente, se compararon por continentes (Europa, Norte América, Oceanía y Asia) las concentraciones medianas de metales pesados de la matriz de revisión, y las observadas en la ciudad de Bogotá y la conurbación de Soacha. La mediana fue utilizada como medida de tendencia central debido a la existencia de valores extremos en los datos de la matriz de revisión bibliográfica desarrollada.

Adicionalmente, se compararon las concentraciones de metales pesados en los hábitats de estudio con respecto a límites legislativos de referencia para la protección de la salud humana en suelo urbano. Se utilizaron los límites establecidos por las siguientes administraciones: (i) Comunidad Autónoma de Cataluña, España (Gencat, 2006); (ii) Comunidad Autónoma del País Vasco, España (Eusko-Legebiltzarra, 2005); y (iii) Canadá (CCME, 1997). Esta legislación fue seleccionada debido a que reportó límites por ingestión, inhalación y contacto dérmico con suelo urbano contaminado por metales pesados. Se calcularon también intervalos de confianza (95\%) para las concentraciones de metales pesados en los hábitats de estudio.

Finalmente, se observaron comparativamente en los hábitats de estudio las concentraciones de metales pesados a partir de los periodos climáticos identificados según la frecuencia de la precipitación (tiempo seco y tiempo de lluvia). Se desarrollaron figuras de concentración de metales pesados en el sedimento vial y de altura de precipitación en relación al tiempo de muestreo, y se determinaron mediante proporciones las diferencias en las concentraciones entre estos dos periodos climáticos. 


\section{Resultados}

\subsection{Análisis comparativo con hábitats urbanos a nivel mundial}

Se realizó una revisión bibliográfica de las concentraciones de metales pesados asociadas con sedimentos viales para diferentes hábitats urbanos a nivel mundial (Tabla 2). La prueba de conglomerados (clúster) permitió la identificación de cuatro racimos: (1) concentraciones de $\mathrm{Zn} \mathrm{y} \mathrm{Cu,} \mathrm{(2)} \mathrm{concentración} \mathrm{de} \mathrm{Cuy}$ año del estudio, (3) área continental y fracción de tamaño del sedimento, (4) área continental y concentración de $\mathrm{Pb}$ (Figura 2). Los racimos 1 y 2 estuvieron agrupados en un nivel superior, posiblemente implicando una relación entre éstos. Igualmente, se evidenció una probable relación de los racimos 3 y 4 . A continuación se presentan los resultados de las relaciones sugeridas por la prueba de conglomerados.

La información presentada en la Tabla 2 mostró que a nivel mundial se reportaron amplias fluctuaciones en las concentraciones de metales pesados asociados con sedimentos viales de hábitats urbanos (Pb: 14-2296 mg/ $\mathrm{kg}$; Zn: $51-2133 \mathrm{mg} / \mathrm{kg}$; Cu: $45-771 \mathrm{mg} / \mathrm{kg}$ ). La prueba de Mann-Whitney mostró que existieron diferencias significativas entre las concentraciones de $\mathrm{Zn} \mathrm{y} \mathrm{Cu}$ (p-valor $<0,001)$. No obstante, esta prueba estadística también mostró que no existieron diferencias significativas entre las concentraciones de $\mathrm{Pb}$ y $\mathrm{Zn}$ ( $\mathrm{p}$-valor $=0,058), \mathrm{y} \mathrm{Pb}$ y Cu (p-valor $=0,230)$. De esta manera, los resultados evidenciaron que las magnitudes de las concentraciones asociadas con el sedimento vial probablemente tuvieron la siguiente secuencia en hábitats urbanos a nivel mundial: $\mathrm{Zn}, \mathrm{Pb}$ y $\mathrm{Cu}$. Es decir, los resultados sugirieron al Zn como el metal pesado más abundante en los sedimentos viales de hábitats urbanos.

Se realizó una comparación entre los anteriores hallazgos detectados nivel mundial y los observados en los hábitats de estudio. Los resultados mostraron que las concentraciones promedio de $\mathrm{Pb}, \mathrm{Zn}$ y $\mathrm{Cu}$ en el sedimento vial de Bogotá y Soacha estuvieron entre $48-217 \mathrm{mg} / \mathrm{kg}$, $96-334 \mathrm{mg} / \mathrm{kg}$ y $41-279 \mathrm{mg} / \mathrm{kg}$, respectivamente (Tabla 3). Esto demostró una tendencia similar a la detectada a nivel mundial, visualizando que los aportes e importancia de las fuentes contaminantes también variaron significativamente en el ambiente vial de Bogotá y Soacha. Adicionalmente, la secuencia en la magnitud de las concentraciones de metales pesados fue similar a la detectada en el ámbito mundial. Es decir, los resultados mostraron en promedio que el $\mathrm{Zn}$ fue el metal pesado más abundante en el ambiente vial de Bogotá y Soacha, seguido de $\mathrm{Cu}$ y $\mathrm{Pb}$. El estudio comparativo de las concentraciones de metales pesados se enfocó sobre la fracción de tamaño inferior a $250 \mu \mathrm{m}$ del sedimento vial, debido a que en la revisión bibliográfica mundial se evidenció que esta fue la tendencia dominante a nivel mundial (Tabla 2).

Figura 2. Dendrograma para el análisis de conglomerados realizado a las variables en estudio (revisión bibliográfica mundial). $\mathrm{AC}=$ Área continental (Europa, Norte América, Oceanía y Asía), FT = Fracción de tamaño del sedimento vial (entre 10-2000 $\mu \mathrm{m}$ ), $\mathrm{Pb}=$ Concentración de plomo $(\mathrm{mg} / \mathrm{kg}), \mathrm{Zn}=$ Concentración de cinc $(\mathrm{mg} / \mathrm{kg})$, $\mathrm{Cu}=$ Concentración de cobre $(\mathrm{mg} / \mathrm{kg})$, y Año = Año del estudio (entre 1982 y 2015)

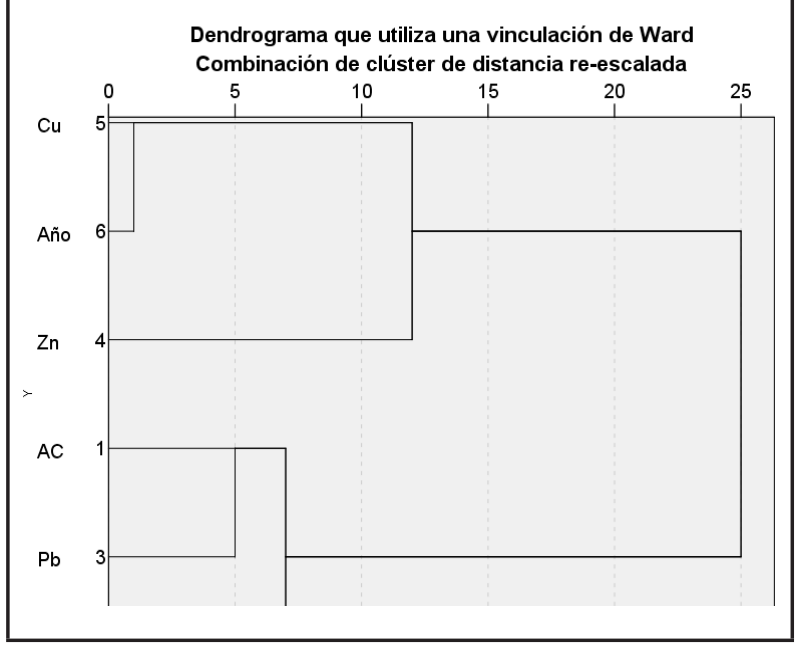

Por otro lado, en la revisión bibliográfica mundial se observó una variabilidad continental en las concentraciones de metales pesados asociadas con los sedimentos viales de hábitats urbanos. Las medianas de las concentraciones de $\mathrm{Pb}, \mathrm{Zn}$ y $\mathrm{Cu}$ en países europeos fueron entre 5\% y 412\% superiores a las registradas en países norteamericanos, siendo las medianas de países asiáticos al menos 59\% superiores en relación a las norteamericanas. En los anteriores análisis la mediana fue utilizada como medida de tendencia central debido a la existencia de valores extremos en la información presentada por la Tabla 2. 
TABLA 2. REVISIÓN BIBLIOGRÁFICA MUNDIAL PARA CONCENTRACIONES DE METALES PESADOS EN SEDIMENTOS VIALES DE HÁBITATS URBANOS. (*) 1 = EUROPA, 2 = NORTE AMÉRICA, $3=$ OCEANÍA, Y $4=$ = ASIA

\begin{tabular}{|c|c|c|c|c|c|c|c|}
\hline \multirow{2}{*}{ Hábitat urbano } & \multirow{2}{*}{$\begin{array}{c}\text { Área } \\
\text { continental } \\
\left({ }^{*}\right)\end{array}$} & \multirow{2}{*}{$\begin{array}{l}\text { Fracción } \\
\text { de tamaño } \\
\qquad(\mu \mathrm{m})\end{array}$} & \multicolumn{3}{|c|}{$\begin{array}{l}\text { Concentración } \\
\text { (mg/kg de materia seca) }\end{array}$} & \multirow[t]{2}{*}{ Año } & \multirow[t]{2}{*}{ Referencia } \\
\hline & & & $\mathrm{Pb}$ & $\mathrm{Zn}$ & $\mathrm{Cu}$ & & \\
\hline London/Inglaterra & 1 & $<250$ & 2296 & 1212 & 386 & 1982 & (Ellis y Revitt, 1982) \\
\hline London/Inglaterra & 1 & $<250$ & 1826 & 695 & 280 & 1982 & (Ellis y Revitt, 1982) \\
\hline London/Inglaterra & 1 & $<250$ & 978 & 2133 & 91 & 1982 & (Ellis y Revitt, 1982) \\
\hline Hildesheim/Alemania & 1 & $<2000$ & 255 & 120 & 84 & 1987 & (Grottker, 1987) \\
\hline Lulea/Suecia & 1 & $75-125$ & 68 & 150 & 89 & 1998 & (Viklander, 1998) \\
\hline Sydney/Australia & 3 & $<200$ & 511 & 249 & 124 & 1998 & (Ball et al., 1998) \\
\hline Lulea/Suecia & 1 & $75-125$ & 15 & 80 & 53 & 1998 & (Viklander, 1998) \\
\hline Lulea/Suecia & 1 & $75-125$ & 14 & 100 & 91 & 1998 & (Viklander, 1998) \\
\hline Jönköping/Suecia & 1 & $<250$ & 45 & 257 & 282 & 2002 & $\begin{array}{c}\text { (German y Svensson, } \\
\text { 2002) }\end{array}$ \\
\hline Jönköping/Suecia & 1 & $<2000$ & 23 & 125 & 119 & 2002 & $\begin{array}{c}\text { (German y Svensson, } \\
\text { 2002) }\end{array}$ \\
\hline $\begin{array}{l}\text { Hamilton/Nueva Zelan- } \\
\text { da }\end{array}$ & 3 & $125-250$ & 251 & 1073 & 184 & 2005 & (Zanders, 2005) \\
\hline Aberdeen/Escocia & 1 & $63-250$ & 305 & 345 & 325 & 2005 & (Deletic y Orr, 2005) \\
\hline Tokyo/Japón & 4 & $<2000$ & 200 & 1300 & 510 & 2008 & (Murakami et al., 2008) \\
\hline Barcelona/España & 1 & $<100$ & 283 & 542 & 216 & 2008 & (Pérez et al., 2008) \\
\hline Barcelona/España & 1 & $<10$ & 229 & 1252 & 771 & 2009 & (Pérez et al., 2008) \\
\hline Zhenjiang/China & 4 & $<2000$ & 589 & 687 & 158 & 2009 & (Bian y Zhu, 2009) \\
\hline Tokyo/Japón & 4 & $<2000$ & - & 1500 & 340 & 2009 & (Murakami et al., 2009) \\
\hline Tokyo/Japón & 4 & $<2000$ & - & 1525 & 708 & 2009 & (Murakami et al., 2009) \\
\hline Baltimore/EE.UU. & 2 & $<63$ & - & 343 & 196 & 2010 & (Helmreich et al., 2010) \\
\hline $\begin{array}{l}\text { Christchurch/Nueva } \\
\text { Zelanda }\end{array}$ & 3 & $<1000$ & 290 & 370 & 73 & 2010 & $\begin{array}{c}\text { (Rijkenberg y Depree, } \\
\text { 2010) }\end{array}$ \\
\hline Beijing/China & 4 & $150-250$ & 59 & 280 & 72 & 2010 & (Zhao et al., 2010) \\
\hline Massachusetts/EE.UU. & 2 & $<2000$ & 79 & 381 & 172 & 2011 & (Apeagyei et al., 2011) \\
\hline Ulsan/Corea del Sur & 4 & $<2000$ & 153 & 325 & 182 & 2011 & (Duong y Lee, 2011) \\
\hline Torrelavega/España & 1 & $125-250$ & 246 & 309 & 90 & 2011 & (Zafra et al., 2011) \\
\hline Torrelavega/España & 1 & $125-250$ & 299 & 309 & 117 & 2011 & (Zafra et al., 2011) \\
\hline Davis/EE.UU. & 2 & $<1000$ & 110 & 414 & 236 & 2012 & $\begin{array}{l}\text { (Kayhanian et al., } \\
\text { 2012) }\end{array}$ \\
\hline Singapore/Malasia & 4 & $<63$ & 297 & 1585 & 465 & 2012 & (Yuen et al., 2012) \\
\hline Bilbao/España & 1 & $<2000$ & 630 & 200 & 45 & 2013 & (Carrero et al., 2013) \\
\hline London/Inglaterra & 1 & $<2000$ & 227 & 1145 & 337 & 2014 & (Crosby et al., 2014) \\
\hline Beijing/China & 4 & $<2000$ & 511 & 51 & 126 & 2015 & (Li et al., 2015) \\
\hline Mediana & & 250 & 251 & 358 & 177 & & \\
\hline Media & & 889 & 400 & 635 & 231 & & \\
\hline Mínimo & & 10 & 14 & 51 & 45 & & \\
\hline Máximo & & 2000 & 2296 & 2133 & 771 & & \\
\hline Datos considerados ( $\mathrm{n}$ ) & & 30 & 27 & 30 & 30 & & \\
\hline
\end{tabular}


Al comparar las concentraciones de metales pesados reportadas a nivel mundial con las observadas en Bogotá y Soacha, se observó que éstas tendieron a ser inferiores en los hábitats colombianos de estudio. Por ejemplo, las concentraciones de $\mathrm{Pb}$ fueron $71 \%, 24 \%$ y $71 \%$ inferiores con respecto a los reportes de vías de Europa, Norteamérica y Asía, respectivamente. Sin embargo, la vía de investigación AA (Soacha, Autopista Sur) registró concentraciones de $\mathrm{Pb}$ 56\% superiores a las reportadas en vías de Norteamérica. Zn y Cu en la vía AA registraron concentraciones inferiores con respecto a todas las áreas continentales en consideración.

Finalmente, se evaluó la concentración de metales pesados para cada vía de Bogotá y Soacha con el objeto de establecer un orden diferenciado en el grado de conta- minación (fracción de tamaño $<250 \mu \mathrm{m}$ ). Los resultados mostraron con respecto al $\mathrm{Pb}$ el siguiente orden: $\mathrm{AA}>\mathrm{SO}$ $>\mathrm{Z} 3>\mathrm{Z1}>\mathrm{Z2}>\mathrm{Z} 4$ (Figura 3). En relación al Zn los resultados fueron los siguientes: $\mathrm{Z} 1>\mathrm{Z} 3>\mathrm{Z} 2>\mathrm{Z} 4>\mathrm{AA}>\mathrm{SO}$. $\mathrm{El} \mathrm{Cu}$ registró una tendencia similar a la reportada para el Zn (Tabla 3). Los resultados mostraron que las vías con concentraciones más elevadas de Pb se localizaron en hábitats de la conurbación de Soacha (AA y SO); mientras que las vías que registraron mayores concentraciones deZn y Cu se localizaron al interior de la ciudad de Bogotá (Z1 y Z3). La fracción de tamaño del sedimento vial inferior a 63 $\mu \mathrm{m}$ presentó resultados similares (Figura 3). No obstante, esta fracción de tamaño registró concentraciones de $\mathrm{Pb}, \mathrm{Zn}$ y Cu 1,23, 1,49 y 1,39 veces más elevadas en relación a la fracción de tamaño inferior a $250 \mu \mathrm{m}$.

TABLA 3. CONCENTRACIÓN PROMEDIO DE METALES PESADOS EN EL SEDIMENTO VIAL DE BOGOTÁ Y SOACHA CON UN 95\% DE INTERVALO DE CONFIANZA. (*) SE RECOLECTARON 36 MUESTRAS DE SEDIMENTO VIAL EN CADA ZONA DE ESTUDIO EN BOGOTÁ:Z1, Z2, Z3, Y Z4 (TOTAL = 144 MUESTRAS); Y SE RECOLECTARON 43 MUESTRAS EN CADA ZONA DE ESTUDIO EN SOACHA: AA Y SO (TOTAL = 86 MUESTRAS). (**) CONCENTRACIONES EN MG/KG DE MATERIA SECA.

\begin{tabular}{|c|c|c|c|c|c|c|c|c|}
\hline $\begin{array}{c}\text { Metal } \\
\left(*^{*}\right)\end{array}$ & $\begin{array}{c}\text { Fontibón } \\
\text { Z1 }\end{array}$ & $\begin{array}{c}\text { Barrios Unidos } \\
\text { Z2 }\end{array}$ & $\begin{array}{c}\text { Kennedy } \\
\text { Z3 }\end{array}$ & $\begin{array}{l}\text { Puente Aranda } \\
\text { Z4 }\end{array}$ & $\begin{array}{c}\text { Autopista Sur } \\
\text { AA }\end{array}$ & $\begin{array}{c}\text { Soacha } \\
\text { SO }\end{array}$ & Promedio & Mediana \\
\hline \multicolumn{9}{|c|}{ Fracción de tamaño $<250 \mu \mathrm{m}$} \\
\hline $\mathrm{Pb}$ & $\left.69 \pm 14^{(* *}\right)$ & $60 \pm 12$ & $74 \pm 15$ & $48 \pm 11$ & $217 \pm 30$ & $84 \pm 22$ & 92 & 72 \\
\hline $\mathrm{Zn}$ & $334 \pm 51$ & $145 \pm 32$ & $197 \pm 29$ & $126 \pm 19$ & $110 \pm 9$ & $96 \pm 13$ & 168 & 136 \\
\hline $\mathrm{Cu}$ & $279 \pm 38$ & $94 \pm 16$ & $110 \pm 19$ & $68 \pm 11$ & $57 \pm 13$ & $41 \pm 10$ & 108 & 81 \\
\hline \multicolumn{9}{|c|}{ Fracción de tamaño $<63 \mu \mathrm{m}$} \\
\hline $\mathrm{Pb}$ & $86 \pm 17$ & $84 \pm 15$ & $88 \pm 19$ & $61 \pm 13$ & $258 \pm 36$ & $99 \pm 26$ & 113 & 87 \\
\hline $\mathrm{Zn}$ & $469 \pm 63$ & $271 \pm 35$ & $301 \pm 48$ & $171 \pm 20$ & $157 \pm 13$ & $137 \pm 19$ & 251 & 221 \\
\hline $\mathrm{Cu}$ & $415 \pm 56$ & $140 \pm 20$ & $141 \pm 22$ & $88 \pm 14$ & $67 \pm 15$ & $46 \pm 11$ & 150 & 114 \\
\hline
\end{tabular}

Figura 3. Concentraciones promedio de metales pesados en los hábitats de estudio (Bogotá y Soacha)
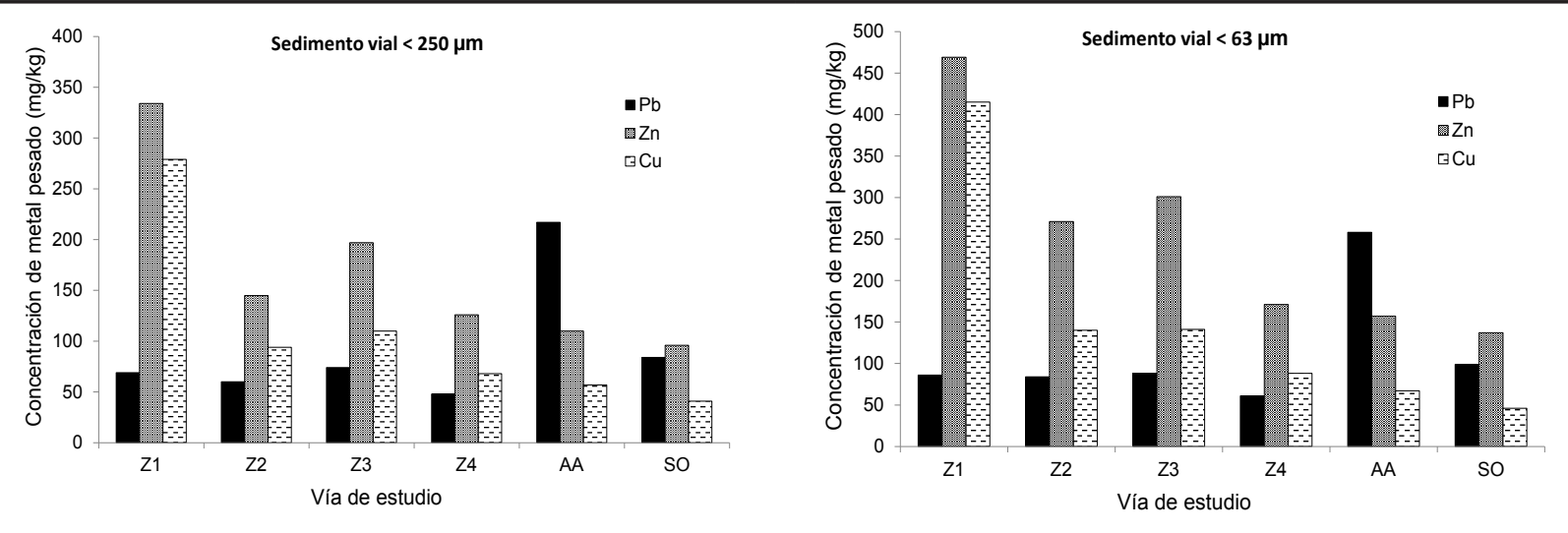


\subsection{Concentraciones locales versus lími-} tes legislativos de referencia

En el presente estudio, el análisis legislativo se centró sobre la fracción de tamaño del sedimento vial inferior a $63 \mu \mathrm{m}$, debido a que ésta registró las mayores concentraciones de metales. Las concentraciones de metales pesados en el sedimento vial fueron comparadas con legislación para la protección de la salud humana (ingestión, inhalación y contacto dérmico) en hábitats urbanos. Se observó que las concentraciones promedio $\mathrm{de} \mathrm{Pb}$ en todas las vías de investigación superaron el límite más exigente de referencia, el establecido por la administración de Cataluña para suelo urbano $(60 \mathrm{mg} /$ $\mathrm{kg}$ ). Con respecto al límite legislativo menos exigente (Canadá = $140 \mathrm{mg} / \mathrm{kg}$; suelo residencial y parques), los resultados mostraron que la vía de estudio en AA fue la única en sobrepasarlo (Tabla 4).

TABLA 4. COMPARACIÓN DE CONCENTRACIONES PROMEDIO DE METALES PESADOS CON RESPECTO A LÍMITES INTERNACIONALES DE REFERENCIA (INTERVALO DE CONFIANZA $=95 \%$; $N=230) .\left({ }^{*}\right)$ NIVELES DE REFERENCIA PARA LA PROTECCIÓN DE LA SALUD HUMANA EN SUELO URBANO (GENCAT, 2006). (**) VALORES INDICATIVOS DE EVALUACIÓN PARA LA PROTECCIÓN DE LA SALUD HUMANA EN SUELO URBANO Y ÁREA DE JUEGO INFANTIL (EUSKO-LEGEBILTZARRA, 2005). $\left.{ }^{* * *}\right)$ VALORES PARA LA PROTECCIÓN DE LA SALUD HUMANA EN SUELO RESIDENCIAL Y PARQUES (CCME, 1997)

\begin{tabular}{|c|c|c|c|}
\hline \multirow{2}{*}{ Vía de investigación } & \multicolumn{3}{|c|}{ Metal pesado (Fracción < $63 \mu \mathrm{m}$ ) } \\
\hline & $\mathrm{Pb}$ & $\mathrm{Zn}$ & $\mathrm{Cu}$ \\
\hline Z1 & $86 \pm 17$ & $469 \pm 63$ & $415 \pm 56$ \\
\hline $\mathrm{Z2}$ & $84 \pm 15$ & $271 \pm 35$ & $140 \pm 20$ \\
\hline $\mathrm{Z3}$ & $88 \pm 19$ & $301 \pm 48$ & $141 \pm 22$ \\
\hline Z4 & $61 \pm 13$ & $171 \pm 20$ & $88 \pm 14$ \\
\hline AA & $258 \pm 36$ & $157 \pm 13$ & $67 \pm 15$ \\
\hline SO & $99 \pm 26$ & $137 \pm 19$ & $46 \pm 11$ \\
\hline Cataluña, España $\left(^{*}\right)$ & 60 & 650 & 310 \\
\hline $\begin{array}{c}\text { País Vasco, España } \\
\qquad(* *)\end{array}$ & 120 & - & - \\
\hline Canadá $(* * *)$ & 140 & 200 & 63 \\
\hline
\end{tabular}

Por otro lado, las concentraciones promedio de Zn en Z1, Z2 y Z3 superaron el límite de referencia más exigente (Canadá $=200 \mathrm{mg} / \mathrm{kg}$; suelo residencial y parques). Sin embargo, todas las vías de estudio no sobrepasaron el límite menos exigente para hábitats urbanos; el establecido por la administración de Cataluña en $650 \mathrm{mg} / \mathrm{kg}$ (Tabla 4). Finalmente, las concentraciones promedio de $\mathrm{Cu}$ superaron en su mayoría el límite legislativo más exigente (Canadá = $63 \mathrm{mg} / \mathrm{kg}$; suelo residencial y parques). Excepto en la vía SO, donde la concentración promedio fue 1,37 veces inferior a dicho límite legislativo. Con respecto al límite de referencia menos exigente para el $\mathrm{Cu}$ (Cataluña $=310 \mathrm{mg} / \mathrm{kg}$; suelo urbano), los resultados mostraron que vía de estudio en Z1 fue la única en sobrepasarlo.

\subsection{Análisis climático para concentracio-} nes de metalles pesados

A partir de información del Instituto de Hidrología, Meteorología y Estudios Ambientales de Colombia (IDEAM) se identificaron los períodos interanuales de incremento de la precipitación en Z1, Z2, Z3 y Z4 para el fenómeno climático de "La Niña-Oscilación del Sur" (García et al., 2012). Estos períodos climáticos correspondieron a tiempo de lluvia, siendo el resto del tiempo de análisis considerado como tiempo seco. El análisis climático no se realizó en la conurbación de Soacha debido a que se contó únicamente con registros para 127 días, lo cual impidió cubrir los periodos climáticos interanuales para la precipitación. En promedio para todos los hábitats de Bogotá, los resultados mostraron que en tiempo seco las concentraciones de $\mathrm{Pb}, \mathrm{Zn}$ y Cu en el sedimento vial $(<63 \mu \mathrm{m})$ fueron comparativamente superiores en relación a las concentraciones observadas en tiempo de lluvia. Por ejemplo, las concentraciones promedio de $\mathrm{Pb}$ en tiempo seco en $\mathrm{Z} 1, \mathrm{Z} 2$, Z3 y Z4 fueron 1,67, 1,01, 1,23, y 1,33 veces superiores en relación a las concentraciones observadas en el sedimento vial en tiempo de lluvia (Tabla 5). La Figura 4 presenta la variación temporal de la concentración $\mathrm{de} \mathrm{Pb}$ en el sedimento vial en los lugares de estudio donde se registraron comparativamente las mayores y menores diferencias entre los periodos climáticos identificados (Z1 y Z3). 


\begin{tabular}{|c|c|c|c|c|c|c|}
\hline \multirow[t]{2}{*}{ Metal } & \multirow[t]{2}{*}{ Vía } & \multicolumn{2}{|c|}{$\begin{array}{l}\text { Concentración }(\mathrm{mg} / \mathrm{kg}) \\
\text { Período climático }\end{array}$} & \multicolumn{3}{|c|}{ Legislación de referencia } \\
\hline & & Seco & Lluvia & $\begin{array}{c}\text { Cataluña, España } \\
\text { (2006) }\end{array}$ & $\begin{array}{l}\text { País Vasco, Espa- } \\
\text { ña }(2005)\end{array}$ & $\begin{array}{c}\text { Canadá } \\
(1997)\end{array}$ \\
\hline \multirow[t]{2}{*}{$\mathrm{Pb}$} & $\begin{array}{l}\mathrm{Z} 1 \\
\mathrm{Z} 2\end{array}$ & $\begin{array}{c}107 \pm 20 \\
84 \pm 13\end{array}$ & $\begin{array}{l}64 \pm 13 \\
83 \pm 16\end{array}$ & \multirow{2}{*}{60} & \multirow{2}{*}{120} & \multirow{2}{*}{140} \\
\hline & $\begin{array}{l}\text { Z3 } \\
\text { Z4 }\end{array}$ & $\begin{array}{l}97 \pm 17 \\
69 \pm 12\end{array}$ & $\begin{array}{l}79 \pm 17 \\
52 \pm 11\end{array}$ & & & \\
\hline \multirow[t]{2}{*}{$\mathrm{Zn}$} & $\begin{array}{l}\mathrm{Z} 1 \\
\mathrm{Z} 2\end{array}$ & $\begin{array}{l}525 \pm 18 \\
298 \pm 12\end{array}$ & $\begin{array}{l}413 \pm 16 \\
244 \pm 12\end{array}$ & \multirow{2}{*}{650} & \multirow{2}{*}{ - } & \multirow{2}{*}{200} \\
\hline & $\begin{array}{l}\mathrm{Z3} \\
\mathrm{Z} 4\end{array}$ & $\begin{array}{c}341 \pm 11 \\
181 \pm 8\end{array}$ & $\begin{array}{c}262 \pm 10 \\
161 \pm 7\end{array}$ & & & \\
\hline \multirow[t]{2}{*}{$\mathrm{Cu}$} & $\begin{array}{l}\mathrm{Z} 1 \\
\mathrm{Z} 2\end{array}$ & $\begin{array}{l}520 \pm 89 \\
309 \pm 47\end{array}$ & $\begin{array}{l}148 \pm 17 \\
132 \pm 22\end{array}$ & \multirow{2}{*}{310} & \multirow{2}{*}{-} & \multirow{2}{*}{63} \\
\hline & $\begin{array}{l}\text { Z3 } \\
\text { Z4 }\end{array}$ & $\begin{array}{l}147 \pm 22 \\
105 \pm 15\end{array}$ & $\begin{array}{l}134 \pm 21 \\
71 \pm 11\end{array}$ & & & \\
\hline
\end{tabular}

Figura 4. Variación temporal de la concentración de Pb en el sedimento vial de Z1 y Z3. Fracción de tamaño $<63$ um. Tiempo seco $=$ A; Tiempo de lluvia $=$ B
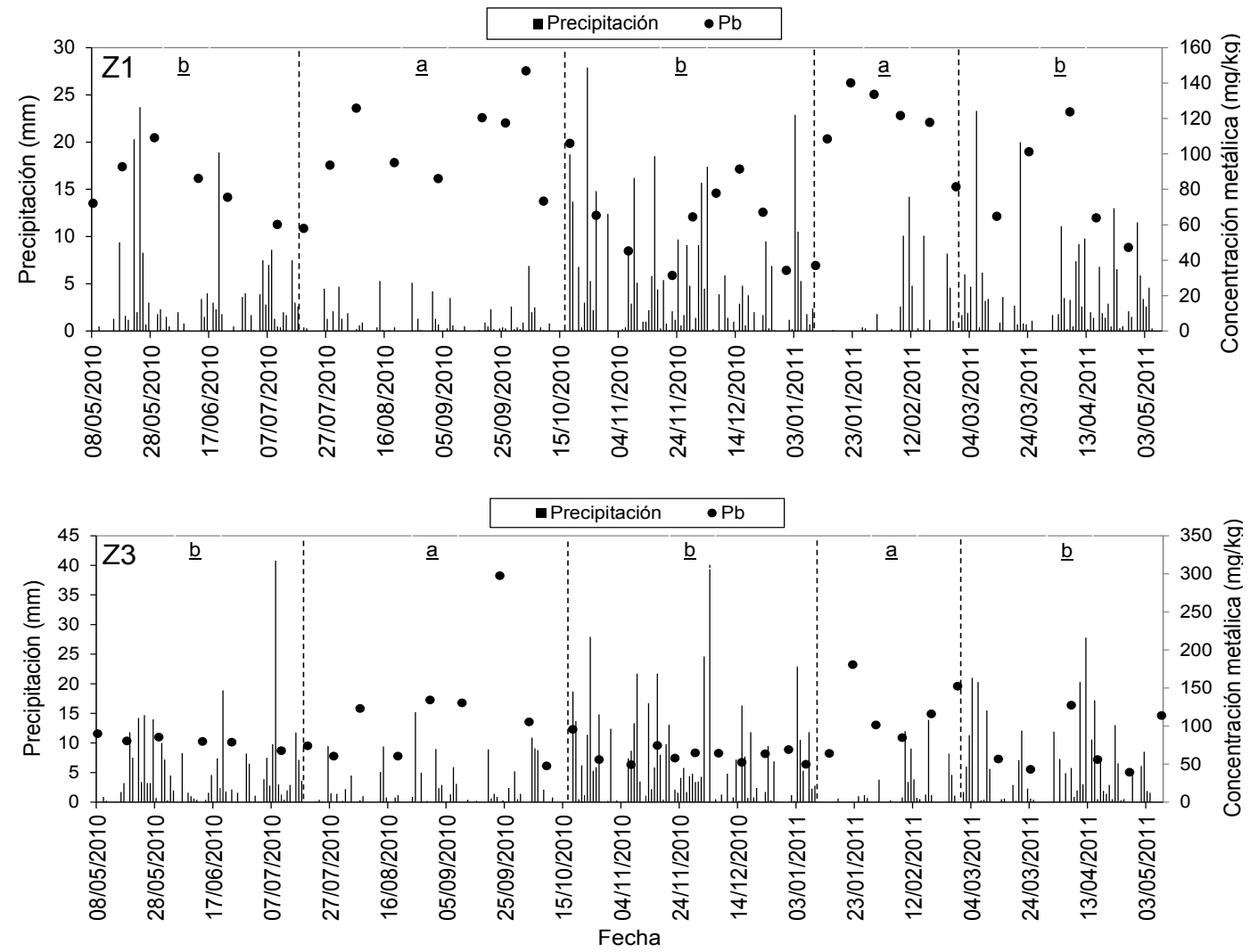


\section{Discusión}

El análisis comparativo con hábitats urbanos a nivel mundial permitió observar una variabilidad continental (Europa, Norte América, Oceanía y Asia) en las concentraciones de metales pesados asociadas con sedimentos viales recolectados en tiempo seco (Tabla 2). Al respecto, el continente asiático tendió a registrar las mayores concentraciones de metales ( $\mathrm{Pb}, \mathrm{Zn}$ y $\mathrm{Cu}$ ). Este incremento en las concentraciones probablemente se debió a que la información asiática correspondió a hábitats densamente urbanizados de ciudades de China (v.g., Zhejiang y Beijing), Japón (Tokio) y Malaysia (Singapur). Kayhanian et al. (2012) reportaron resultados similares para concentraciones de metales pesados $(\mathrm{Pb}, \mathrm{Zn}$ y $\mathrm{Cu}$ ) en el agua de escorrentía vial de hábitats urbanos a nivel mundial.

Adicionalmente, existió coincidencia entre las concentraciones de metales reportadas a nivel mundial y las observadas en Bogotá y Soacha para $\mathrm{Pb}$, Zn y Cu. Es decir, los resultados sugirieron al Zn como el elemento metálico más abundante en los sedimentos viales de los hábitats urbanos en estudio $(\mathrm{Zn}=168 \mathrm{mg} / \mathrm{kg}$; $\mathrm{Cu}$ = $108 \mathrm{mg} / \mathrm{kg} ; \mathrm{Pb}=92 \mathrm{mg} / \mathrm{kg}$ ). Sin embargo, en la vía AA el metal más abundante fue el $\mathrm{Pb}$ (Tabla 3). Este incremento en la concentración de Pb se debió probablemente a que este hábitat presentó el mayor tráfico promedio diario (40100 vehículos/día). Los resultados también insinuaron que los aportes e importancia de las fuentes de metales pesados (fijas: industrias; y móviles: vehículos) probablemente variaron significativamente en las vías de los hábitats en estudio. Lo anterior debido al rango observado en las concentraciones de metales pesados (Pb: 48-217 mg/kg, Zn: 96-334 mg/kg, y Cu: $41-279 \mathrm{mg} / \mathrm{kg}$ ).

El análisis comparativo con hábitats urbanos a nivel mundial también permitió observar que las concentraciones de metales pesados en el sedimento vial de Bogotá tendieron a ser inferiores en relación a las reportadas en vías de Norteamérica, Europa y Asía entre los años 1982 y 2015. Sin embargo, la vía de investigación AA en Soacha registró una concentración promedio de $\mathrm{Pb}$ 2,28 veces superior a la reportada en vías de Norteamérica. Esta última área continental fue la que registró las menores concentraciones de $\mathrm{Pb}$ en el ámbito mundial. El incremento en la concentración de Pb en la vía AA probablemente estuvo asociado con el elevado tráfico promedio diario observado en este hábitat de estudio (40100 vehículos/día), en comparación con el tráfico vehicular observado en las demás vías de Bogotá y Soacha (entre 650 y 13500 vehículos/día). Por lo tanto, los resultados evidenciaron que las vías en Soacha (AA y SO) registraron las mayores concentraciones de $\mathrm{Pb}$; mientras que las vías en Bogotá (Z1 y Z3) registraron las mayores concentraciones de $\mathrm{Zn}$ y $\mathrm{Cu}$. El anterior análisis se centró en la fracción de tamaño menor de $250 \mu \mathrm{m}$ del sedimento vial, debido a que ésta fue reportada a nivel mundial como la fracción representativa del contenido metálico en hábitats urbanos (Tabla 2).

Los resultados evidenciaron que la fracción más fina del sedimento vial $(<63 \mu \mathrm{m})$ tendió a registrar las mayores concentraciones de metales pesados en los hábitats urbanos de estudio. En promedio, esta fracción de tamaño presentó una concentración de metales pesados 1,37 veces superior en relación a la fracción de tamaño menor de $250 \mu \mathrm{m}$ (Tabla 3). De esta manera, los resultados sugirieron un incremento en la concentración de metales pesados a medida que disminuyó el tamaño de partícula del sedimento vial. Esta tendencia probablemente se debió a que las fuentes de metales pesados en los hábitats de estudio emitieron tamaños de partícula asociados con la fracción más fina del sedimento vial. Esta fracción fina también ha sido reportada como la de mayor potencial de inhalación, ingestión y contacto dérmico por parte de los residentes de hábitat urbanos, el cual fue intensificado por la resuspensión del sedimento vial a causa de altas velocidades del viento y la turbulencia del tráfico (Amato et al., 2013).

A partir de lo anterior, la comparación con límites legislativos de referencia se realizó para la fracción de tamaño más fina del sedimento vial $(<63 \mu \mathrm{m})$. La legislación de referencia tuvo en cuenta la inhalación, ingestión y contacto dérmico con suelo de hábitats urbanos contaminados con metales pesados; específicamente, para suelos residenciales, áreas de juego infantil y parques públicos. Existió evidencia que el elemento más crítico en Bogotá y Soacha fue el $\mathrm{Pb}$. En promedio, los resultados evidenciaron que las concentraciones de $\mathrm{Pb}$ superaron entre 1,02 y 4,30 veces el límite de referencia más exigente: Cataluña, España $=60 \mathrm{mg} /$ $\mathrm{kg}$ (Gencat, 2006). Por lo tanto, la vía localizada en AA (Soacha Autopista Sur) requiere de especial atención 
debido a que mostró las concentraciones más elevadas de $\mathrm{Pb}$ (promedio $=258 \mathrm{mg} / \mathrm{kg}$ ) en relación a los demás hábitats de estudio. Con respecto a los restantes metales pesados, la comparación con límites legislativos evidenció que las estrategias de control para la protección de los hábitats de estudio deberían enfocarse inicialmente en el $\mathrm{Cu}$ antes que en el $\mathrm{Zn}$.

El análisis climático permitió evidenciar la existencia de variaciones en las concentraciones de metales pesados durante el período de observación en Bogotá. Estas variaciones probablemente estuvieron asociadas con el régimen de precipitación evidenciado sobre los hábitats urbanos de estudio. Es decir, los resultados sugirieron al periodo de tiempo seco (disminución de la precipitación) como el de mayor atención por parte de las autoridades ambientales y de salud pública, debido a que se observaron incrementos en las concentraciones de metales pesados asociadas con el sedimento vial. En promedio, las concentraciones de $\mathrm{Pb}, \mathrm{Zn}$ y $\mathrm{Cu}$ durante este período de tiempo seco fueron 1,31, 1,23 y 2,11 veces superiores en relación a las observadas en tiempo de lluvia (incremento de la precipitación), respectivamente.

Como era de esperar, los resultados evidenciaron que durante los períodos de tiempo seco existió una mayor excedencia en las concentraciones de metales pesados en relación a los límites legislativos de referencia. En promedio, durante estos períodos de tiempo existieron excedencias para $\mathrm{Pb}$ en todas las vías de estudio en Bogotá; entre 1,78 y 1,15 veces en relación al límite legislativo más exigente: Cataluña, España $=60 \mathrm{mg} /$ $\mathrm{kg}$ (Gencat, 2006). En tiempo de lluvia las excedencias fueron menores, entre 1,38 y 1,07 veces. Excepto en Z4, donde no se superó este límite legislativo de referencia (Tabla 5). Romero et al. (2015) reportaron una tendencia similar en relación a la influencia de la precipitación y la escorrentía superficial sobre las concentraciones de metales pesados en sedimentos viales de hábitats urbanos. Estos hallazgos permitieron sugerir un aporte de metales pesados en sistemas hídricos por parte de los sedimentos viales urbanos. Finalmente, Schaller et al. (2016) reportaron la influencia del fenómeno climático de Oscilación del Sur-El Niño (OSEN) en el régimen y magnitud de las precipitaciones y la escorrentía superficial de hábitats urbanos latinoamericanos.

\section{Conclusiones}

Los resultados sugieren al Zn como el metal pesado más abundante en los sedimentos viales de los hábitats urbanos en estudio. No obstante, el elemento metálico más crítico en el marco de la salud pública urbana es el $\mathrm{Pb}$. Existe evidencia de un incremento en la concentración de este metal pesado cuando se presenta un elevado tráfico vehicular. Los hallazgos también muestran que durante los periodos climáticos de tiempo seco (disminución de la precipitación) las autoridades ambientales y de salud pública deben implementar medidas de vigilancia y control más estrictas, debido a que se observan incrementos en las concentraciones de metales pesados asociadas con el sedimento vial.

Por lo tanto, esta investigación se constituye en un punto de referencia para el desarrollo de estrategias en el marco del urbanismo, el ambiente y la salud pública, en relación a la contaminación generada por los metales pesados asociados con sedimentos viales; como por ejemplo, la implementación de restricciones en el uso del suelo según el grado de contaminación, la reducción del tráfico vehicular en hábitats de elevada contaminación, y el incremento de la frecuencia del barrido vial en tiempo seco. Esta investigación también es útil para la discusión y publicación de legislación para la protección de la salud humana y ambiental en megaciudades latinoamericanas de características similares.

Sin embargo, las siguientes limitaciones forman parte de este estudio y requieren de especial atención. (i) Las superficies viales seleccionadas no presentan características similares en relación a fuentes fijas y móviles de emisión de metales pesados (v.g., cantidad y tipo de industrias, y composición e intensidad del tráfico). La influencia de estos factores locales probablemente debe ser evaluada en detalle para estudios futuros sobre la variación espacial de las concentraciones de metales pesados en hábitats urbanos. (ii) En el estudio de influencia de la precipitación deberían considerarse factores adicionales como por ejemplo la intensidad, frecuencia y duración de las lluvias, periodo seco previo, pendiente vial, y rugosidad del pavimento; los cuales podrían influir en la disminución o incremento de las concentraciones de metales pesados asociados con sedimentos viales de hábitats urbanos. 


\section{Referencias}

Amato, F., Schaap, M., Denier van der Gon, H.A.C., Pandolfi, M., Alastuey, A., y Keuken, M. (2013). Short-term variability of mineral dust, metals and carbon emission from road dust resuspension. Atmospheric Environment, 74, pp. 34-40. doi: https://doi.org/10.1016/j. atmosenv.2013.03.037.

Apeagyei, E., Bank, M.S., y Spengler, J.D. (2011). Distribution of heavy metals in road dust along an urban-rural gradient in Massachusetts. Atmospheric Environment, 45(13), pp. 2310-2323. doi: https://doi.org/10.1016/j. atmosenv.2010.11.015

Arcas-Abella, J., Pagès-Ramon, A., y Casals-Tres, M. (2011). El futuro del hábitat: repensando la habitabilidad desde la sostenibilidad. El caso español. Revista INVI, 26(72), pp. 65-93. doi: http://dx.doi.org/10.4067/ S0718-83582011000200003.

Ball, J.E., Jenks, R., y Aubourg, D. (1998). An assessment of the availability of pollutant constituents on road surfaces. Science of the Total Environment, 209(2-3), pp. 243-254. doi: https://doi.org/10.1016/S00489697(98)80115-0.

Bian, B., y Zhu, W. (2009). Particle size distribution and pollutants in road-deposited sediments in different areas of Zhenjiang, China. Environmental Geochemistry and Health, 31(4), pp. 511-20. doi: https://doi.org/10.1007/ s10653-008-9203-8.

Carrero, J.A., Arrizabalaga, I., Bustamante, J., Goienaga, N., Arana, G., y Madariaga, J.M. (2013). Diagnosing the traffic impact on roadside soils through a multianalytical data analysis of the concentration profiles of traffic-related elements. Science of the Total Environment, pp. 458-460, 427-434. doi: https://doi. org/10.1016/j.scitotenv.2013.04.047.

CCME, Canadian Council of Ministers of the Environment. (1997). Recommended Canadian soil quality guidelines. (1a. ed.). Winnipeg, Canada: CCME Press.

Crosby, C.J., Fullen, M.A., Booth, C.A., y Searle, D.E. (2014). A dynamic approach to urban road deposited sediment pollution monitoring (Marylebone Road, London, UK). Journal of Applied Geophysics, 105, pp. 10-20. doi: https://doi.org/10.1016/j.jappgeo.2014.03.006.

Deletic, A., y Orr, D.W. (2005). Pollution buildup on road surfaces. Journal of Environmental Engineering, 131(1), pp. 49-59. doi: https://doi.org/10.1061/(ASCE)07339372(2005)131:1(49).
Dockery, D., y Pope, A. (1994). Epidemiology of acute health effects: summary of time series studies. Cambridge, USA: Harvard University Press.

Duong, T.T.T., y Lee, B. (2011). Determining contamination level of heavy metals in road dust from busy traffic areas with different characteristics. Journal of Environmental Management, 92(3), pp. 554-562. doi: https:// doi.org/10.1016/j.jenvman.2010.09.010.

Ellis, J.B., y Revitt, D.M. (1982). Incidence of heavy metals in street surface sediments: Solubility and grain size studies. Water, Air and Soil Pollution, 17(1), pp. 87-100. doi: https://doi.org/10.1007/BF00164094.

Eriksson, E., Baun, A., Scholes, L., Ledin, A., Ahlman, S., y Revitt, M. (2007). Selected stormwater priority pollutants - a European perspective. Science of the Total Environment, 383(1-3), pp. 41-51. doi: https://doi. org/10.1016/j.scitotenv.2007.05.028.

Eusko-Legebiltzarra. (2005). Ley 1/2005: Prevención de la contaminación del suelo del País Vasco. (1a. ed.). Bilbao, España: Boletín Oficial del País Vasco.

García, M.C., Piñeros, A., Bernal, F.A., y Ardila, E. (2012). Climate Variability, Climate Change and Water Resources in Colombia. Revista de Ingeniería, 36, pp. 60-64. Disponible en: http://www.scielo.org.co/pdf/ ring/n36/n36a12.pdf

Gencat, Generalitat de Catalunya. (2006). Niveles genéricos de referencia (NGR), valores de los NGR para metales y metaloides y protección de la salud humana aplicables a Cataluña. (1a. ed.). Barcelona, España: Agencia de Residuos de Cataluña.

German, J., y Svensson, G. (2002). Metal content and particle size distribution of street sediments and street sweeping waste. Water Science Technology, 46(6-7), pp. 191-198.

Grottker, M. (1987). Runoff quality from a street with medium traffic loading. Science of the Total Environment, 59(C), pp. 457-466. doi: https://doi.org/10.1016/00489697(87)90469-4.

Helmreich, B., Hilliges, R., Schriewer, A., y Horn, H. (2010). Runoff pollutants of a highly trafficked urban road - Correlation analysis and seasonal influences. Chemosphere, 80(9), pp. 991-997. doi: https://doi. org/10.1016/j.chemosphere.2010.05.037.

ISO, International Organization for Standardization. Standards Handbook. (1a. ed.). Geneva, Switzerland: ISO Press. 
Kayhanian, M., McKenzie, E.R., Leatherbarrow, J.E., y Young, T.M. (2012). Characteristics of road sediment fractionated particles captured from paved surfaces, surface run-off and detention basins. Science of the Total Environment, 439, pp. 172-186. doi: https://doi. org/10.1016/j.scitotenv.2012.08.077.

Li, H., Shi, A., y Zhang, X. (2015). Particle size distribution and characteristics of heavy metals in road-deposited sediments from Beijing Olympic Park. Journal of Environmental Sciences, 32, pp. 228-237. doi: https://doi. org/10.1016/j.jes.2014.11.014.

Liu, E., Yan, T., Birch, G., y Zhu, Y. (2014). Pollution and health risk of potentially toxic metals in urban road dust in Nanjing, a mega-city of China. Science of the Total Environment, 476-477, pp. 522-531. doi: https:// doi.org/10.1016/j.scitotenv.2014.01.055.

Murakami, M., Fujita, M., Furumai, H., Kasuga, I., y Kurisu, F. (2009). Sorption behavior of heavy metal species by soakaway sediment receiving urban road runoff from residential and heavily trafficked areas. Journal of Hazardous Materials, 164(2-3), pp. 707-712. doi: https://doi.org/10.1016/j.jhazmat.2008.08.052.

Murakami, M., Nakajima, F., y Furumai, H. (2008). The sorption of heavy metal species by sediments in soakaways receiving urban road runoff. Chemosphere, 70(11), pp. 2099-2109. doi: https://doi.org/10.1016/j. chemosphere.2007.08.073.

Nelson, G., Maisonet, M., y Miyashiro, M. (2005). Evaluación de los efectos de la Contaminación del aire en la Salud de América Latina y El Caribe. Washington, USA: Organización Panamericana de la Salud.

Pérez, G., López-Mesas, M., y Valiente, M. (2008). Assessment of heavy metals remobilization by fractionation: Comparison of leaching tests applied to roadside sediments. Environmental Science and Technology, 42(7), pp. 2309-2315. doi: https://doi.org/10.1021/ es0712975.

Qing, X., Yutong, Z., y Shenggao, L. (2015). Assessment of heavy metal pollution and human health risk in urban soils of steel industrial city (Anshan), Liaoning, northeast China. Ecotoxicology and Environmental Safety, 120, pp. 377-385. doi: https://doi.org/10.1016/j. ecoenv.2015.06.019.

Rijkenberg, M.J.A., y Depree, C.V. (2010). Heavy metal stabilization in contaminated road-derived sediments. Science of the Total Environment, 408(5), pp. 1212-1220. doi: https://doi.org/10.1016/j.scitotenv.2009.11.053.
Romero-Barreiro, M.P., Pinilla-Castañeda, R.D., y ZafraMejía, C.A. (2015). Temporal assessment of the heavy metals $(\mathrm{Pb}$ and $\mathrm{Cu})$ concentration associated with the road sediment: Fontibón-Barrios Unidos (Bogotá D.C., Colombia). Ingeniería y Universidad, 19(2), pp. 315-333. doi: http://dx.doi.org/10.1114/javeriana. iyu19-2.etcm.

Sarmiento, R., Hernández, L.J., Medina, E.K., Rodríguez, N., y Reyes, J. (2015). Respiratory symptoms associated with air pollution in five localities of Bogotá, 20082011, a dynamic cohort study. Biomédica, 2015, 35(3), pp.167-176.doi: http://dx.doi.org/10.7705/biomedica. v35i0.2445.

Schaller, S., Jean-Baptiste, N., \& Lehmann, P. (2016). Opportunities and barriers for urban adaptation to climate change in Latin America. Cases of Mexico City, Lima and Santiago de Chile. Eure, 42(127), pp. 257-278. doi: http://dx.doi.org/10.4067/S025071612016000300011.

SDA, Secretaría Distrital de Ambiente de Bogotá. Informe anual calidad del aire de Bogotá, año 2011. (1ª. ed.). Bogotá, Colombia: Alcaldía Mayor de Bogotá; 2012.

Vera, A.M., Venegas, A.M., Pertuz-Cruz, S.L., y Angulo, R. (2015). An analysis of environmental determinants regarding the safety of vegetables farmed and consumed in rural areas around Tenjo, Colombia. Revista de la Facultad de Medicina, 63(1), pp. 57-68. doi: http:// dx.doi.org/10.15446/revfacmed.v63n1.41098.

Viklander, M. (1998). Particle size distribution and metal content in street sediments. Journal of Environmental Engineering, 124(8), pp. 761-6. doi: https://doi. org/10.1061/(ASCE)0733-9372(1998)124:8(761).

Yuen, J.Q., Olin, P.H., Lim, H.S., Benner, S.G., Sutherland, R.A., y Ziegler, A.D. (2012). Accumulation of potentially toxic elements in road deposited sediments in residential and light industrial neighborhoods of Singapore. Journal of Environmental Management, 101, pp. 151-163. doi: https://doi.org/10.1016/j.jenvman.2011.11.017.

Zafra, C.A., Temprano, J., y Tejero, I. (2011). Distribution of the concentration of heavy metals associated with the sediment particles accumulated on road surfaces. Environmental Technology, 32(9), pp. 997-1008. doi: http://dx.doi.org/10.1080/09593330.2010.523436.

Zafra, C., Temprano, J., y Tejero, I. (2017). The physical factors affecting heavy metals accumulated in the sediment deposited on road surfaces in dry weather: A review. Urban Water Journal, 14(6), pp. 639-649. doi: https://doi.org/10.1080/1573062X.2016.1223320 
Zanders, J.M. (2005) Road sediment: Characterization and implications for the performance of vegetated strips for treating road run-off. Science of the Total Environment, 339(1-3), pp. 41-47. doi: https://doi. org/10.1016/j.scitotenv.2004.07.023.

Zhao, H., Li, X., Wang, X., y Tian, D. (2010). Grain size distribution of road-deposited sediment and its contribution to heavy metal pollution in urban runoff in Beijing, China. Journal of Hazardous Materials, 183(1-3), pp. 203-210. doi: https://doi.org/10.1016/j. jhazmat.2010.07.012.

Zafra-Mejía, C.; Beltrán-Vargas, J.; Hernández-Peña, Y. (2020). Contaminación y salud pública en hábitats urbanos: metales pesados en sedimentos viales de Bogotá, Colombia. Revista EIA, 17(33) enero-junio, Reia33001, pág 1-15. Disponible en: https://doi.org/10.24050/reia.v17i33.1247 